\title{
EL CINE Y LA PRENSA ESCRITA COMO MEDIOS DE INNOVACIÓN DOCENTE EN LA ENSEÑANZA DEL DERECHO PROCESAL
}

\author{
THE CINEMA AND THE PRESS WRITTEN AS MEDIA OF TEACHING \\ INNOVATION IN THE TEACHING OF PROCEDURAL LAW
}

\author{
RUBÉN LÓPEZ PICÓ \\ Becario de Formación Interna en Derecho Procesal \\ rubenlopezpico@ugr.es \\ Universidad de Granada
}

\begin{abstract}
Resumen:
El presente trabajo describe dos actividades de innovación docente que se desarrollarán durante el curso académico 2018-2019 en la asignatura Delincuencia Juvenil -grupo A, único- del Grado en Criminología y en la asignatura de Derecho Procesal II -grupos A y B- del Grado en Derecho y Ciencias Políticas, ambos de la Universidad de Granada. El desarrollo de estas dos actividades de innovación docente consistirá en la utilización del cine y de la prensa escrita como instrumentos innovadores en la enseñanza del Derecho Procesal; como recursos docente para que los alumnos de las asignaturas antes señaladas, además, de profundizar y reforzar los conocimientos teóricos adquiridos, desarrollen las habilidades y competencias propias de la materia. El empleo de estas dos herramientas en la enseñanza del Derecho Procesal permite establecer el inicio o el hilo conductor -dependiendo del momento- en la explicación y posterior debate sobre una institución o cuestión procesal, al tiempo, que incidir sobre la motivación del alumnado $\mathrm{y}$, en consecuencia, sobre su aprendizaje.
\end{abstract}

Palabras clave: Innovación, Docencia, Metodología docente, Enseñanza del derecho, Aprendizaje innovador, Cine, Prensa escrita.

\begin{abstract}
:
The present work describes two activities of teaching innovation that will be developed during the academic year 2018-2019 in the subject Youth Delinquency -group A, onlyof the Degree in Criminology and in the subject of Procedural Law II -groups A and Bof the Degree in Law and Political Science, both from the University of Granada. The development of these two teaching innovation activities will consist in the use of the cinema and the written press as innovative instruments in the teaching of Procedural Law; as teaching resources so that the students of the previously mentioned subjects, in addition, to deepen and reinforce the theoretical knowledge acquired, develop the skills and competences of the subject. The use of these two tools in the teaching of Procedural Law allows establishing the beginning or the thread -depending on the moment- in the explanation and subsequent debate on an institution or procedural question, at the time, that affect the motivation of the students and, consequently, about their learning.
\end{abstract}

REJIE Nueva época: Revista Jurídica de Investigación e Innovación Educativa

Núm.19, Enero 2019, pp. 25-38

[En línea] http://www.revistas.uma.es/index.php/rejie

Recibido: julio 2018

Aceptado: octubre 2018 
Keywords: Innovation, Teaching, Teaching methodology, Teaching of law, Innovative learning, Cinema, Press.

Sumario: 1. Introducción. 2. Objetivos. 3. El cine como medio de innovación docente en la enseñanza del Derecho Procesal. 3.1. Metodología. 3.2. Desarrollo de la actividad de innovación docente. 3.3. Evaluación. 4. La prensa escrita como medio de innovación docente en la enseñanza del Derecho Procesal. 4.1. Metodología. 4.2. Desarrollo de la actividad de innovación docente. 4.3. Evaluación. 5. Reflexiones finales. Bibliografía.

\section{Introducción.}

Las dos experiencias de innovación docente que se presentan serán llevadas a cabo, respectivamente, en la docencia práctica correspondiente a la asignatura Delincuencia Juvenil del Grado en Criminología y Derecho Procesal II del Doble Grado en Derecho y Ciencias Políticas, respectivamente, ambas de la Universidad de Granada durante el curso académico 2018-2019.

La asignatura Delincuencia Juvenil del Grado en Criminología de la Universidad de Granada es una asignatura de carácter obligatorio y semestral de 6 créditos ECTS -la parte correspondiente al proceso penal de menores y, por ende, la impartida por el profesorado del Departamento de Derecho Procesal y Eclesiástico del Estado de la Universidad de Granada es de 3 créditos- que se imparte en el primer semestre de $4^{\circ}$ curso -grupo A, único- de dicha titulación bajo la dirección del profesor GARRIDO CARRILLO $^{1}$. Las clases de esta asignatura serán impartidas todos los lunes y martes en horario de $16 \mathrm{~h}$ a $18 \mathrm{~h}$ durante un espacio de tiempo de 15 semanas a razón 4 horas/semana.

Por su parte, la asignatura Derecho Procesal II del Doble Grado en Derecho y Ciencias Políticas de la Universidad de Granada también es una asignatura de carácter obligatorio y semestral de 8 créditos ECTS, que en el caso concreto de esta titulación Doble Grado en Derecho y Ciencias Políticas- se imparte durante el segundo semestre de $3^{\circ}$ curso -grupos A y B- bajo la supervisión del profesor GONZÁLEZ PALMERO ${ }^{2}$. Las clases de esta asignatura se desarrollarán durante todos los miércoles, jueves y viernes -en horario de $11 \mathrm{~h}$ a $13 \mathrm{~h}$ para el grupo A; y de $18 \mathrm{~h}$ a $20 \mathrm{~h}$ para el grupo Bdurante un periodo temporal de 5 semanas a razón de 6 horas/semana y de 10 semanas a razón de 5 horas/semana.

Por medio de estas dos experiencias de innovación docente, pretendemos sustituir el enfoque tradicionalmente atribuido a la docencia práctica de ambas asignaturas consistente en la resolución por los estudiantes de una serie de supuestos prácticos y en la contestación escrita a las cuestiones planteadas por el profesorado de la asignatura mediante el uso de la legislación, jurisprudencia y bibliografía- por una dinámica completamente nueva basada en la utilización del cine y de la prensa escrita como medios e instrumentos docentes, prácticos e innovadores en la enseñanza y el

\footnotetext{
${ }^{1}$ D. Francisco Javier Garrido Carrillo. Profesor Titular de Derecho Procesal de la Universidad de Granada.

${ }^{2}$ D. Francisco González Palmero. Profesor de Derecho Procesal de la Universidad de Granada. Abogado.
} 
aprendizaje -respectivamente- del Derecho Procesal. A través del desarrollo de las distintas sesiones -tanto de cine como de prensa-, los alumnos ponen en práctica los conocimientos jurídicos adquiridos de forma previa, durante el desarrollo de las clases teóricas -clases magistrales-; desarrollando, de ese modo, las competencias y capacidades básicas de la práctica del Derecho Procesal.

Mi participación en la docencia práctica de ambas asignaturas durante el curso académico 2018-2019 será posible tras haberme sido concedida la Venia Docendi bajo la tutela y supervisión de los profesores antes señalados. Será entonces cuando pondremos en práctica estas dos experiencias de innovación docente. Si los resultados obtenidos con su desarrollo son los esperados, estas dos actividades de innovación docente volverán a ser implementadas en el próximo curso académico -2019/2020-, siendo mejoradas cuando así necesario y en lo que sea necesario.

\section{Objetivos.}

El nuevo modelo educativo European Credit Transfer System -ECTS- impuesto por el Espacio Europeo de Educación Superior -EEES-, centrado en el aprendizaje autónomo de los alumnos bajo la supervisión -tutorización- del profesorado universitario responsable de las diferentes asignaturas -Delincuencia Juvenil y Derecho Procesal II, en nuestro caso-, lleva aparejado consigo la necesidad de repensar las tradicionales actividades de enseñanza y aprendizaje y su sustitución por el empleo de herramientas docentes realmente útiles en la elaboración, adquisición y transmisión de los conocimientos necesarios dentro del nuevo modelo educativo en el que estamos inmersos ${ }^{3}$.

Conscientes de esta nueva realidad y, por consiguiente, de este nuevo reto, defendemos la necesidad de que el profesorado universitario experimente un cambio radical con respecto al papel o rol que desempeña, al menos en lo que al proceso de enseñanza y aprendizaje se refiere. Debiendo apostar para ello, por el empleo de herramientas docentes que, de carácter accesorio a las tradicionales, permitan llevar a cabo el desarrollo de innovadores métodos pedagógicos.

Simultáneamente, junto a las nuevas exigencias educativas impuestas por el EEES, nuestra preocupación por que los alumnos universitarios, además de las propias de la materia impartida -Delincuencia Juvenil y Derecho Procesal, respectivamente, en nuestro caso concreto-, adquieran las denominadas competencias genéricas -trabajo cooperativo, capacidades de comunicación y experiencia profesional, entre otras muchas- que, en un futuro próximo, les faciliten su incorporación al mercado laboral, nos lleva a tenerlas presente en el momento de confeccionar los nuevos y modernos métodos de enseñanza y aprendizaje a los que antes nos hemos referido ${ }^{4}$.

\footnotetext{
${ }^{3}$ GARRIDO CARRILLO, F.J. (2012), "La orientación y tutoría académica, profesional y personal del estudiante de Derecho", en GARRIDO CARRILLO, F.J.: Intercambios y buenas prácticas en la enseñanza del Derecho. Nuevos Métodos Docentes. Granada: Comares.

${ }^{4}$ LÓPEZ PICÓ, R. (2018), "El empleo del cine como medio de innovación en la enseñanza del Derecho Procesal", en MENOR CAMPOS, D.J., PINEDA MARTOS, C., BERLANGA CAÑETE, F.A., RAYA BERMÚDEZ, A.I. y LEIVA CANDIA, D.E.: Libro resúmenes de comunicaciones. I Congreso Virtual Internacional de Innovación Docente Universitaria. Córdoba: Córdoba University Press.
} 
En este mismo sentido, el empleo del cine y la prensa escrita en la asignatura Delincuencia Juvenil y Derecho Procesal II -respectivamente-, persiguen -como medios de innovación docente para la enseñanza del Derecho Procesal- la consecución de los siguientes objetivos:

a) La adquisición de los conocimientos técnico-jurídicos necesarios para el posterior desarrollo de la praxis jurídica.

b) La utilización de nuevos instrumentos docentes para la enseñanza práctica del Derecho Procesal.

c) Complementar los conocimientos teóricos y manejo de la bibliografía, legislación y jurisprudencia, con el análisis de casos reales y supuestos, que permitan configurar en tiempo real situaciones jurídicas diferencias que requieran la aplicación de los conocimientos jurídicos adquiridos con anterioridad.

d) Mejorar la capacidad de análisis y síntesis por los estudiantes de las situaciones jurídicas propuestas.

e) El desarrollo por los estudiantes de un espíritu crítico personal, y de las habilidades relativas a la argumentación jurídica, exposición y al diálogo en público.

f) La potenciación de la motivación y el autoaprendizaje de los estudiantes, profundizando en la aplicabilidad práctica de los conocimientos jurídicos que previamente han adquirido en clase.

g) Mejora de las capacidades de trabajo en grupo.

h) La adquisición y el ejercicio por los estudiantes de competencias diversas: competencias instrumentales -capacidad de análisis y síntesis; capacidad de gestión de la información; y capacidad de toma de decisiones y resolución de problemas-, competencias interpersonales -capacidad de crítica y autocritica; capacidad de apreciación y respeto de la diversidad; y compromiso ético y profesional-, y competencias sistemáticas -capacidad para aplicar los conocimientos jurídicos adquiridos; capacidad para la investigación; $y$ capacidad para el autoaprendizaje-.

\section{El cine como medio de innovación docente en la enseñanza del Derecho Procesal.}

Las primeras experiencias relacionadas con el empleo del cine como medio de innovación docente en la enseñanza del Derecho Procesal, tuvieron lugar en la Universidad de Granada bajo la dirección del profesor GARRIDO CARRILLO ${ }^{5}$.

\footnotetext{
${ }^{5}$ El profesor GARRIDO CARRILLO, forma parte, desde su constitución, de la sección de Derecho Procesal integrada dentro de la Red de Profesores para la elaboración de Materiales Didácticos para la enseñanza del derecho a través del cine. En la actualidad, esta Red se encuentra coordinada por el profesor FERREIRO BAAMONDE -Profesor Titular de Derecho Procesal de la Universidad de Coruña-. En relación a esta misma cuestión, también debemos señalar que el profesor GARRIDO CARRILLO ha sido el Director de los distintos ciclos de Derecho y Cine "Fotogramas Jurídicos" que a lo largo de los últimos años han tenido lugar en la Facultad de Derecho de la Universidad de Granada.
} 
A través del empleo del cine como medio de innovación docente en la enseñanza del Derecho Procesal durante el desarrollo de las clases encuadradas dentro de la docencia práctica de algunas de las asignaturas impartidas por el Departamento de Derecho Procesal y Eclesiástico del Estado de la Universidad de Granada, el profesor GARRIDO CARRILLO pretendía que sus estudiantes reforzasen y profundizasen en el estudio y la comprensión de determinados conceptos jurídicos de carácter procesal esenciales para el posterior desarrollo de la práctica jurídica $\mathrm{y}$, por ende, de sus futuras actividades profesionales.

Y es que tal y como afirma CÁMARA RUIZ ${ }^{6}$ "los nuevos modelos de enseñanza no pueden obviar una realidad indiscutible: la imagen ha superado al texto escrito. Como primera vía de acercamiento a una materia, es más fácil captar la atención de cualquier alumno con la proyección de una película que con una exposición de diapositivas repletas de esquemas y comentarios escritos en un ámbito tan específico como el jurídico, caracterizado por una vertiente teórica muy marcada. Los códigos y los manuales priman como herramienta básica para el estudio de cualquier materia, sin embargo, esta propuesta de estudiar instituciones, principios y conceptos jurídicos de Derecho Procesal a través de películas supone un paso más en el desarrollo de nuevas metodologías que favorezcan el aprendizaje completo a través de la aplicación práctica".

\subsection{Metodología.}

La asignatura Delincuencia Juvenil del Grado en Criminología de la Universidad de Granada se compone de un número de estudiantes no excesivamente elevado, lo que permite que todos los estudiantes puedan visualizar la película y participar en el posterior debate que se dirige sobre la misma en una única sesión, sin necesidad de esperas o de la realización y organización de turnos de trabajo.

En otro orden de cosas, señalar que los materiales necesarios para el desarrollo de esta actividad de innovación docente se reducen a las películas que se pretenden proyectar "Matar a un ruiseñor" 7 dirigida por Robert Mulligan, por medio de la cual se trata de extraer consecuencias referidas al principio de igualdad de partes. Sobre todo desde la perspectiva de la igualdad real frente a la igualdad formal; "El crimen de Cuenca" ${ }^{8}$ dirigida por Pilar Miró, recoge la cuestión relativa al error judicial y a los problemas que en el sistema acusatorio actual existen para alcanzar la verdad y, por ende, si la presunción de inocencia, el principio in dubio pro reo y las normas del proceso penal sobre la carga de la prueba son o no suficientes para garantizar la justicia del resultado;

\footnotetext{
${ }^{6}$ CÁRAMA RUIZ, J. (2011), "Herramientas y técnicas para la docencia del Derecho Procesal”, en PICÓ I JUNOY, J.: El aprendizaje del Derecho Procesal. Nuevos retos de la enseñanza universitaria. Barcelona: Bosch.

${ }^{7}$ FERREIRO BAAMONDE, X. (2009), "Matar un reiseñor o dereito a un proceso xusto", en PERNAS GARCÍA, J.J. (Coord.): $O$ ensino do Dereito a través do cinema: unha perspectiva interdisciplinar. Materiais didácticos para un sistema ECTS. Coruña: Universidad de Coruña, Universidad de Santiago, y Universidad de Vigo.

${ }^{8}$ CUBILlO LÓPEZ, I.J., FERNÁNDEZ CARRÓN, C., y DE PRADA RODRÍGUEZ, M. (2011), "Utilización del cine como herramienta para la docencia del Derecho Procesal", en PICÓ I JUNOY, J.: Aprendizaje del Derecho Procesal. Nuevos retos en la enseñanza universitaria. Barcelona: Bosch.
} 
"12 hombres sin piedad" dirigida por Sidney Lumet, aborda la institución de carácter procesal penal del Jurado. Utilizada para reflexionar sobre la conveniencia de la propia institución, el sistema de elección de sus miembros y la deliberación de éstos últimos, entre otras muchas cuestiones- y a la ficha técnica de éstas -a la que ya antes hemos hecho referencia-. Por otro lado, indicar que esta actividad de innovación docente se desarrollará en la Sala de Vistas de la Facultad de Derecho de la Universidad de Granada o en una de las Aulas polivalentes ubicadas en el Aulario de Derecho de la Universidad de Granada que disponen de todos los medios audiovisuales necesarios para la proyección de las películas y, como resultado, para el desarrollo de la actividad de innovación docente.

La realización de esta actividad de innovación docente tendrá lugar dentro de las horas de clases computables a la parte de la docencia práctica de la asignatura, debiendo reservarse al menos 3 sesiones prácticas -6 horas- para su realización. Al tener las clases una duración de 2 horas, estimamos oportunos distribuir el tiempo de la siguiente forma: proyección de la película - 1 h y 30 minutos- y debate posterior -30 minutos-. La realización de esta misma actividad de innovación docente tendrá lugar durante todo el semestre de forma coordinada con las explicaciones teóricas del contenido de la asignatura impartidas por el profesorado responsable de la misma, correspondientes a la parte de la docencia teórica de la asignatura.

\subsection{Desarrollo de la actividad de innovación docente.}

El desarrollo de esta actividad de innovación docente, consistente en la utilización del cine como medio de innovación docente en la enseñanza del Derecho Procesal, requiere en primer lugar la selección de las diferentes películas -nacionales e internacionalesque se van a proyectar. La correcta selección, el enfoque y la presentación de las películas seleccionadas constituye un elemento básico para el éxito de esta actividad de innovación docente, de ahí, la importancia de que las películas seleccionadas aborden de una forma correcta y centrada las diferentes instituciones y los conceptos de Derecho Procesal que se desean $\operatorname{trabajar}^{10}$.

Así, la primera parte de esta actividad de innovación docente consistirá en la presentación de la película y la institución o el concepto de Derecho Procesal a trabajar, haciendo el profesorado responsable de la asignatura y, por ende, de esta actividad de innovación docente, la correspondiente explicación e indicación de cuáles son los elementos claves que servirán de referencia para los alumnos; no siendo otros que los que constituyen la dimensión jurídica de la película que se va a proyectar. En la segunda parte de esta misma actividad de innovación docente se proyectará la película previamente seleccionada y en la tercera se abrirá un debate entre los propios alumnos en torno a la institución o concepto de Derecho Procesal objeto de análisis.

\footnotetext{
${ }^{9}$ DE PRADA RODRÍGUEZ, M. (2013), "El aprendizaje del derecho procesal a través del cine", en BERZOSA LÓPEZ, D., CUADRADO PÉREZ, C., CALATAYUD PRATS, I., y VELASCO FABRA, G.J. (Coord.).: La evaluación e innovación docente en el Grado de Derecho. Cizur Menor: Aranzadi. ${ }^{10}$ PICÓ I JUNOY, J., ADÁN DOMÉNECH, F, CERRATO GURI, E., y CASANOVA MARTÍ, R. (2015), "El cine como metodología docente del derecho procesal", en VILLCA POZO, M., y CARRERAS I CASANOVAS, A.: Docencia virtual y experiencias de innovación docente: entornos blearning y e-learning. Barcelona: Huygens, D.L.
} 
Por último, y de forma posterior al desarrollo de la actividad de innovación docente, los alumnos deberán cumplimentar -en el plazo máximo de quince días- una ficha técnica, respondiendo a las distintas preguntas en ella formuladas y elaborar un comentario jurídico de carácter crítico de la película proyectada. En dicho comentario jurídico, los alumnos de forma argumentada y reflexionada deberán establecer los puntos de conexión existentes entre la institución o el concepto jurídico de Derecho Procesal indicado por el profesorado de la asignatura y el contenido de la película proyectada en la clase.

Inspirados en el empleo del cine como instrumento metodológico por los profesores FERREIRO BAAMONDE y PÉREZ-CRUZ MARTÍN ${ }^{11}$, con el fin de poder orientar y guiar a los alumnos en la realización del comentario jurídico de carácter crítico de la película proyectada en clase, el profesorado les entregará una ficha técnica formada por varios apartados que deberán de completar:

1. Datos artísticos y técnicos de la película proyectada.

2. Breve descripción de la temática jurídica de la película, a modo de sinopsis argumental.

3. Comentario jurídico propio de la película proyectada realizado por el estudiante.

4. Resolución por los estudiantes de las distintas preguntas planteadas por el profesorado. Presenta como finalidad esencial la de comprobar la adquisición por los estudiantes de los conocimientos jurídicos pretendidos al iniciar la actividad. Las preguntas variarán dependiendo de los conceptos jurídicos que se pretenden reforzar a través del visionado de la correspondiente película.

5. Búsqueda por los estudiantes de películas y libros cuya temática esté relacionada con la temática de la película proyectada en clase.

Cabe también articular una variante para enriquecer el debate generado de forma posterior a la proyección de la película seleccionada: la organización de los alumnos en grupos dependiendo del rol jurídico que más les interese. De tal manera, que tanto en el visionado de la película, como en el posterior debate y en la realización de la ficha técnica, se trabaje por grupos y se fijen posiciones comunes y contrapuestas variables en función del rol jurídico previamente adoptado ${ }^{12}$.

\subsection{Evaluación.}

Recordar que la asignatura Delincuencia Juvenil -grupo A, único- del Grado en Criminología de la Universidad de Granada se compone de 6 créditos, de los cuales 3 créditos recaen sobre el Departamento de Derecho Procesal y Eclesiástico del Estado de

\footnotetext{
${ }^{11}$ FERREIRO BAAMONDE, X. y PÉREZ-CRUZ MARTÍN, A.J. (2011), "El cine como instrumento metodológico en la docencia del Derecho Procesal", en PICÓ I JUNOY, J.: El aprendizaje del Derecho Procesal. Nuevos retos de la enseñanza universitaria. Barcelona: Bosch.

${ }^{12}$ GARRIDO CARRILLO, F.J. (2012), "Los espacios virtuales interactivos -EVI- en la enseñanza del Derecho: un modelo para la docencia de Derecho Procesal", en GARRIDO CARRILLO, F.J.: Intercambios y buenas prácticas en la enseñanza del Derecho. Nuevos Métodos Docentes. Granada: Comares.
} 
la Universidad de Granada. Con la intención de poder impartir el contenido teórico de la asignatura al tiempo que desarrollar la actividad de innovación docente propuesta, tal y como antes hemos señalado, de las 30 horas -3 créditos- de las se disponen, solo 6 horas $-0,6$ créditos- serán destinadas al desarrollo de esta actividad de innovación docente. Las 24 horas restantes $-2,4$ créditos- se destinarán a la explicación del contenido teórico de la asignatura a través de las clases magistrales impartidas por el profesorado universitario responsable de ella.

Conforme al contenido de la Guía Docente y Didáctica de la asignatura Delincuencia Juvenil -grupo A, único- del Grado en Criminología de la Universidad de Granada, su calificación final estará formada, de un lado, por la nota obtenida en el examen final que los alumnos realicen de la asignatura $-70 \%$ de la nota final de la asignatura-, y de otro lado, por la calificación que se obtenga por la realización del resto de actividades $-30 \%$ de la calificación final de la asignatura. De ese modo, la participación de los alumnos en el desarrollo de esta actividad de innovación docente podrá ser valorada con hasta un máximo de 3 puntos -1 punto por cada una de las tres películas que se pretenden proyectar-. En el momento de calificar la actividad realizada por el alumno se valorará esencialmente su participación activa en el debate generado de forma posterior a la proyección de la película y la calidad del comentario jurídico crítico que de la película proyectada en clase haya realizado.

La participación de los alumnos en el desarrollo de esta actividad de innovación docente será plenamente voluntaria, pero sobreentendiendo que, salvo por razones debidamente justificadas y comunicadas con anterioridad al inicio de la misma al profesorado de la asignatura que impidiesen al alumno participar en ella, todos los alumnos matriculados en la asignatura Delincuencia Juvenil -grupo A, único- del Grado en Criminología de la Universidad de Granada participarán en su desarrollo. En todo caso, para poder superar positivamente la asignatura será necesario que el alumno apruebe el examen final -escrito u oral- de la misma.

\section{La prensa escrita como medio de innovación docente en la enseñanza del Derecho Procesal.}

La enseñanza y aprendizaje de instituciones y conceptos jurídicos de Derecho Procesal a través del empleo de noticias o sucesos de carácter jurídico recogidos en la prensa local, autonómica y nacional -tanto escrita como digital-, despierta -como resultado de su veracidad, proximidad y popularidad- el interés y la implicación de los alumnos en la fase de investigación, como en la de recopilación de información y en la aplicación de los conocimientos de Derecho Procesal previamente adquiridos en clase a los hechos descritos y narrados en ella. Potenciándose de este modo, la motivación y el interés de los alumnos ${ }^{13}$.

\footnotetext{
${ }^{13}$ GRANDE SEARA, P. y ESPINO HERNÁNDEZ, D. (2011), "La prensa escrita como recurso para el aprendizaje del Derecho Procesal Penal”, en PICÓ I JUNOY, J.: El aprendizaje del Derecho Procesal. Nuevos retos de la enseñanza universitaria. Barcelona: Bosch. En el mismo sentido, SERRANO MORENO J.L. (Coord.) (2014). Cine y literatura como herramientas didácticas. Granada: Godel.
} 


\subsection{Metodología.}

En el caso concreto del Doble Grado en Derecho y Ciencia Políticas de la Universidad de Granada, la asignatura Derecho Procesal II está compuesta por un número de alumnos -tanto el grupo A como el B- no excesivamente elevado, lo que permite que los alumnos puedan trabajar de forma individual y simultánea, sin necesidad de tener que constituir grupos de trabajo que acaben por ralentizar el desarrollo de la actividad de innovación docente a la par que generar desequilibrios con respecto al grado de implicación y al trabajo realizado por cada uno de los distintos miembros que componen los diferentes grupos de trabajo. Ante un supuesto contrario al que acabamos de describir, recomendamos la formación de grupos de trabajo reducidos -4 o 5 alumnos, como máximo- para evitar, precisamente, los desequilibrios con respecto al grado de implicación y al trabajo realizado por cada uno de los distintos miembros que componen los diferentes grupos de trabajo, a los que antes nos hemos referido.

El principal inconveniente que se podría plantear a la hora de llevar a cabo el desarrollo de esta actividad de innovación docente radicaría en el hecho de que como bien hemos dicho antes, sus destinatarios son los alumnos de $3^{\circ}$ curso -grupo A y B- del Doble Grado en Derecho y Ciencias Políticas de la Universidad de Granada, que podrían carecer de los conocimientos jurídicos necesarios para poder participar en su desarrollo. Sin embargo, frente a este posible argumento, entendemos y presuponemos que todos los alumnos que se encuentran matriculados en la asignatura de Derecho Procesal II grupo A y B- perteneciente al $3^{\circ}$ curso del Doble Grado en Derecho y Ciencias Políticas de la Universidad de Granada, ya disponen de todos los conocimientos jurídicos que son necesarios para poder participar en el desarrollo de la misma. Máxime cuando en los cursos académicos anteriores, ya han debido de cursar y superar satisfactoriamente la asignatura Derecho Procesal I -de carácter obligatorio-, así como otras asignaturas de carácter optativo cuya docencia también es impartida por el Departamento de Derecho Procesal y Eclesiástico del Estado de la Universidad de Granada -Arbitraje y Procesos Civiles, Proceso Contencioso Administrativo, Procesos Laborales, y Procesos Constitucionales-.

En cuanto a los materiales necesarios para el desarrollo de esta actividad de innovación docente, debemos hacer referencia a los diferentes diarios informativos de carácter impreso y digital -nacionales: El Mundo, el País, ABC, o La Razón; y locales: Ideal de Granada, Granada Hoy, o Granada Ciudad-. Se recomienda la selección de sucesos acaecidos a nivel local o autonómico, en los que el seguimiento informativo de los mismos es mayor- ${ }^{14}$, la legislación, la bibliografía y la jurisprudencia, así como otros textos legales. La Facultad Derecho de la Universidad de Granada dispone de todos los medios materiales -biblioteca, aula de informática, seminarios, bases de datos, etc.- que son necesarios para el correcto desarrollo de esta actividad de innovación docente, quedando siempre y en todo momento a disposición del profesorado y de los alumnos ${ }^{15}$.

\footnotetext{
${ }^{14}$ GARCÍA MARTÍN, R. (2014), “Análisis crítico de actualidad jurídica como método docente”, Reduca (Derecho). Serie Derecho Procesal, vol.5, núm.1, pp.18-29.

${ }^{15}$ GARRIDO CARRILLO, F.J. y ARTACHO MARTÍN-LAGOS, M. (2012), "Nuevas tecnologías y adquisición de competencias en la enseñanza del Derecho", en GARRIDO CARRILLO, F.J.: Intercambios y buenas prácticas en la enseñanza del Derecho. Nuevos Métodos Docentes. Granada: Comares.
} 
La realización de esta actividad de innovación docente tendrá lugar dentro de las horas de clases computables a la parte de la docencia práctica de la asignatura, debiendo reservarse al menos 5 sesiones prácticas -10 horas- para su realización. La realización de esta misma actividad de innovación docente tendrá lugar a lo largo de todo el semestre coordinadamente con las explicaciones del contenido teórico de la asignatura impartidas -a través de las clases magistrales- por el profesorado responsable de la misma, dentro de la parte de la docencia teórica de la asignatura. Lo afirmado constituye una mera previsión, pues la realización de la actividad de innovación docente quedará en todo momento supeditado al tiempo disponible, así como a la propia organización docente de la asignatura.

\subsection{Desarrollo de la actividad de innovación docente.}

El desarrollo de esta segunda actividad de innovación docente, consistente en la utilización de la prensa escrita como instrumento en la enseñanza del Derecho Procesal, requiere de la búsqueda en los diferentes diarios informativos -nacionales y locales- de noticias jurídicas cuyo contenido en materia de Derecho Procesal penal sea consistente y, por ende, apto para el desarrollo de la misma. La correcta realización de esta primera tarea resulta primordial para la consecución del objetivo principal propuesto -la enseñanza del Derecho Procesal a través de la utilización de la prensa escrita como medio de innovación docente- y de todos los demás objetivos también antes señalados segundo apartado: "Objetivos"-. Es por ello reseñable, el enorme interés en la localización y el uso de noticias que referidas a un mismo asunto, difieran en el tiempo y permitan realizar un seguimiento de las distintas cuestiones y aspectos procesales que en el pudiesen observarse con relación a cada una de las fases del proceso penal. El análisis de esas mismas noticias desde diferentes líneas editoriales enriquecerá en gran medida el desarrollo de esta actividad de innovación docente ${ }^{16}$.

El desarrollo de esta actividad de innovación docente estará dividido en cinco grandes sesiones:

1. La primera sesión servirá para informar y explicar a los alumnos en qué va a consistir el desarrollo de ésta actividad de innovación docente. Ya en esa primera sesión se establecerán los contenidos teóricos del programa de la asignatura que se pretenden trabajar a través de ella, así como las pautas y orientaciones necesarias para su desarrollo. También se aclararán las posibles dudas que pudiesen existir sobre la propia actividad de innovación docente, sobre su desarrollo o sobre su evolución, y se establecerá el cronograma relativo a su desarrollo.

2. La segunda sesión se dividirá en dos partes. En la primera de ellas, los alumnos tendrán que trabajar autónomamente y desde sus casas. Así, durante los 15 días posteriores a la celebración de la primera sesión, los alumnos deberán buscar la noticia de prensa -de carácter nacional o local relacionada con el contenido de la asignatura- que utilizarán para el desarrollo de toda la actividad. Se les

16 FONT RIBAS, A. (2004), "Líneas maestras en el aprendizaje por problemas", Revista Interuniversitaria de Formación del Profesorado, núm. 49, pp.79-96. 
recomendará que a la hora de escoger la noticia, intenten que ésta cuente con el mayor número de elementos posibles relacionados con el contenido teórico de la asignatura y, a ser posible, que sea una noticia de Derecho Procesal penal. Pues aunque el contenido de la asignatura también cuenta con una parte de Derecho Procesal civil, la parte de Derecho Procesal penal es la predominante en ella. Será de enorme interés establecer temas guías como por ejemplo: las cuestiones sobre la orden europea de detención -Caso Puigdemont-; las diligencias previas e investigación de un crimen -Caso de Diana Quer-; o la prueba en el juicio oral -Caso "La manada"-.

Durante ese mismo especio de tiempo, y antes de iniciar la segunda parte de esta misma sesión, los alumnos deberán haber recopilado todas las noticias de prensa relacionadas con el caso jurídico que hayan seleccionado, leerlas y confrontarlas, a fin de establecer una relación de los hechos jurídicos más destacados, especialmente de aquellos que estén relacionados con el contenido teórico de la asignatura.

Una vez ya en clase, durante la segunda parte de esta sesión, los alumnos realizarán una exposición pública de todo el trabajo realizado hasta el momento, y responderán a las posibles preguntas que el profesorado o el resto de los alumnos les puedan plantear sobre los distintos aspectos procesales de los hechos jurídicos noticiables seleccionados.

3. Cuando en las clases teóricas impartidas por el profesorado de la asignatura se hayan abordado los temas relativos a la denuncia, la querella y las partes acusadoras en el proceso penal, se iniciará el desarrollo de la tercera sesión. En ella, cada alumno redactará una denuncia partiendo de los hechos jurídicos de los que se componen la noticia elegida, que tras ser entregada al profesor, deberá ser expuesta públicamente en clase.

4. Explicados en clase los temas relativos a la detención y a la prisión preventiva, se desarrollará la cuarta sesión. Ésta se centrará en el estudio de las diferentes cuestiones relativas a la detención de los imputados, la prisión provisional y su duración, la libertad provisional bajo fianza, y el cómputo posible de la condena.

5. La quinta y última de las sesiones de la que se compone el desarrollo de esta actividad de innovación docente se destinará a practicar la redacción de los escritos que correspondan en atención a la fase procedimental en la que se encuentre el hecho judicial objeto de la noticia.

De forma común a todas las sesiones, cabe como variable que el resto de los alumnos conformen grupos que asuman diferentes roles procesales -Juzgador, Acusación Particular, Acusación Popular, Ministerio Fiscal, Defensa- para desde éstos poder debatir las distintas cuestiones procesales relacionadas con cada una de las noticias previamente seleccionadas ${ }^{17}$.

\footnotetext{
${ }^{17}$ CABALlOL I ANGELATS, L. (2011), "La docencia del Derecho Procesal basado en problemas", en PICÓ I JUNOY, J.: El aprendizaje del Derecho Procesal. Nuevos retos de la enseñanza universitaria. Barcelona: Bosch.
} 


\subsection{Evaluación.}

Nuevamente, recordar que la asignatura Derecho Procesal II -grupo A y B- del Doble Grado en Derecho y Ciencias Políticas de la Universidad de Granada se compone de 6 créditos. Con la intención de poder impartir el contenido teórico de la asignatura al tiempo que desarrollar la actividad de innovación docente propuesta, tal y como antes hemos señalado, de las 60 horas -6 créditos- de las se disponen, solo 10 horas -1 créditos- serán destinadas al desarrollo de esta actividad de innovación docente. Las 50 horas restantes -5 créditos- se destinarán a la explicación del contenido teórico de la asignatura a través de las clases magistrales impartidas por el profesorado universitario responsable de ella y, por ende, de la esta actividad de innovación docente.

De acuerdo al contenido de la Guía Docente y Didáctica de la asignatura Derecho Procesal II -grupo A y B- del Doble Grado en Derecho y Ciencias Políticas de la Universidad de Granada, su calificación final se compondrá, de un lado, por la nota obtenida en el examen final que los alumnos realicen de la asignatura $-70 \%$ de la nota final de la asignatura-, y de otro lado, por la calificación que se obtenga por la realización del resto de actividades $-30 \%$ de la calificación final de la asignatura. Así, la participación de los alumnos en el desarrollo de esta actividad de innovación docente podrá ser valorada con hasta un máximo de 3 puntos.

La participación de los alumnos en el desarrollo de esta actividad de innovación docente será plenamente voluntaria, pero sobreentendiendo que, salvo por razones debidamente justificadas y comunicadas con anterioridad al inicio de la misma al profesorado de la asignatura que impidiesen al alumno participar en ella, todos los alumnos matriculados en la asignatura Derecho Procesal II -grupo A y B- del Doble Grado en Derecho y Ciencias Políticas de la Universidad de Granada participarán en su desarrollo. En todo caso, para poder superar positivamente la asignatura será necesario que el alumno apruebe el examen final -escrito u oral- de la misma.

\section{Reflexiones finales.}

A través de la participación de los alumnos en el desarrollo de estas dos actividades de innovación docente, se pretende que éstos profundicen y refuercen el estudio de determinadas instituciones y conceptos jurídicos de Derecho Procesal esenciales para el posterior desarrollo de la práctica jurídica $\mathrm{y}$, por ende, de sus futuras actividades profesionales; al tiempo, que pongan en práctica el conjunto de conocimientos jurídicos de Derecho Procesal que previamente han adquirido en clase a través de las lecciones magistrales impartidas por el profesorado responsable de ambas asignaturas. Objetivos que antes, por medio de los tradicionales sistemas de enseñanza basados en las clases magistrales de la asignatura impartidas por el profesorado universitario y el desarrollo de ciertas prácticas basadas en la contestación por el alumnado a determinadas cuestiones planteadas por el profesorado, eran imposibles de alcanzar.

Mientras los alumnos se convierten en los auténticos protagonistas de estas dos actividades de innovación docente, al constatar como los conocimientos jurídicos que previamente han adquiridos en las clases teóricas impartidas por el profesorado de la 
asignatura pueden ser aplicados directamente en la práctica jurídica -a través de una película o de un caso real recogido en prensa, respectivamente-; el papel desempeñado por el profesorado responsable de las asignaturas y, como resultado, de estas dos actividades de innovación docente, durante el desarrollo de las mismas, no es el de un mero espectador, sino más bien todo lo contrario. El profesorado interactúa constantemente con los estudiantes: participando en el debate que se genera de forma posterior a la proyección de la película, planteando diferentes cuestiones, resolviendo las dudas que pudiesen invadir a los alumnos y facilitándoles a éstos últimos los materiales que les son necesarios para su participación en el desarrollo de ambas actividades de innovación docente. Es por ello, que el éxito o el fracaso de estas dos actividades de innovación docente dependerán en gran medida del nivel de interacción alcanzado entre el profesorado y los alumnos que en ellas participan.

Entendemos que trabajar con estos supuestos -películas y casos jurídicos recogidos en prensa, respectivamente- despierta el interés, la implicación y la motivación de los alumnos; transformando las clases prácticas de la asignatura en una actividad dinámica y atractiva para ellos, así como en una oportunidad para potenciar y fomentar su capacidad crítica y de autoaprendizaje.

\section{Bibliografía.}

-CABALLOL I ANGELATS, L. (2011), "La docencia del Derecho Procesal basado en problemas", en PICÓ I JUNOY, J.: El aprendizaje del Derecho Procesal. Nuevos retos de la enseñanza universitaria. Barcelona: Bosch.

-CÁRAMA RUIZ, J. (2011), "Herramientas y técnicas para la docencia del Derecho Procesal”, en PICÓ I JUNOY, J.: El aprendizaje del Derecho Procesal. Nuevos retos de la enseñanza universitaria. Barcelona: Bosch.

-CUBILLO LÓPEZ, I.J., FERNÁNDEZ CARRÓN, C., y DE PRADA RODRÍGUEZ, M. (2011), "Utilización del cine como herramienta para la docencia del Derecho Procesal", en PICÓ I JUNOY, J.: Aprendizaje del Derecho Procesal. Nuevos retos en la enseñanza universitaria. Barcelona: Bosch.

-DE PRADA RODRÍGUEZ, M. (2013), “El aprendizaje del derecho procesal a través del cine", en BERZOSA LÓPEZ, D., CUADRADO PÉREZ, C., CALATAYUD PRATS, I., y VELASCO FABRA, G.J. (Coord.).: La evaluación e innovación docente en el Grado de Derecho. Cizur Menor: Aranzadi.

-FERREIRO BAAMONDE, X. (2009), "Matar un reiseñor o dereito a un proceso xusto", en PERNAS GARCÍA, J.J. (Coord.).: O ensino do Dereito a través do cinema: unha perspectiva interdisciplinar. Materiais didácticos para un sistema ECTS. Coruña: Universidad de Coruña, Universidad de Santiago, y Universidad de Vigo. 
-FERREIRO BAAMONDE, X. y PÉREZ-CRUZ MARTÍN, A.J. (2011), "El cine como instrumento metodológico en la docencia del Derecho Procesal", en PICÓ I JUNOY, J.: El aprendizaje del Derecho Procesal. Nuevos retos de la enseñanza universitaria. Barcelona: Bosch.

-FONT RIBAS, A. (2004), "Líneas maestras en el aprendizaje por problemas", Revista Interuniversitaria de Formación del Profesorado, núm. 49, pp.79-96.

-GARCÍA MARTÍN, R. (2014), "Análisis crítico de actualidad jurídica como método docente”, Reduca (Derecho). Serie Derecho Procesal, vol.5, núm.1, pp.18-29.

-GARRIDO CARRILLO, F.J. (2012), "La orientación y tutoría académica, profesional y personal del estudiante de Derecho", en GARRIDO CARRILLO, F.J.: Intercambios y buenas prácticas en la enseñanza del Derecho. Nuevos Métodos Docentes. Granada: Comares.

-GARRIDO CARRILLO, F.J. y ARTACHO MARTÍN-LAGOS, M. (2012), "Nuevas tecnologías y adquisición de competencias en la enseñanza del Derecho", en GARRIDO CARRILlO, F.J.: Intercambios y buenas prácticas en la enseñanza del Derecho. Nuevos Métodos Docentes. Granada: Comares.

-GARRIDO CARRILLO, F.J. (2012), "Los espacios virtuales interactivos (EVI) en la enseñanza del Derecho: un modelo para la docencia de Derecho Procesal", en GARRIDO CARRILLO, F.J.: Intercambios y buenas prácticas en la enseñanza del Derecho. Nuevos Métodos Docentes. Granada: Comares.

-GRANDE SEARA, P. y ESPINO HERNÁNDEZ, D. (2011), "La prensa escrita como recurso para el aprendizaje del Derecho Procesal Penal", en PICÓ I JUNOY, J.: El aprendizaje del Derecho Procesal. Nuevos retos de la enseñanza universitaria. Barcelona: Bosch.

-LÓPEZ PICÓ, R. (2018), "El empleo del cine como medio de innovación en la enseñanza del Derecho Procesal", en MENOR CAMPOS, D.J., PINEDA MARTOS, C., BERLANGA CAÑETE, F.A., RAYA BERMÚDEZ, A.I. y LEIVA CANDIA, D.E.: Libro resúmenes de comunicaciones. I Congreso Virtual Internacional de Innovación Docente Universitaria. Córdoba: Córdoba University Press.

-PICÓ I JUNOY, J., ADÁN DOMÉNECH, F, CERRATO GURI, E., y CASANOVA MARTÍ, R. (2015), "El cine como metodología docente del derecho procesal", en VILLCA POZO, M., y CARRERAS I CASANOVAS, A.: Docencia virtual y experiencias de innovación docente: entornos b-learning y e-learning. Barcelona: Huygens, D.L.

-SERRANO MORENO J.L. (Coord.) (2014). Cine y literatura como herramientas didácticas. Granada: Godel. 